\title{
THE EXPLOITATION OF WEBGIS BASED ON ARCGIS SERVER AND AJAX
}

\author{
Xue Lei ${ }^{1}$, Li Lin ${ }^{*}$, Longhe Wang ${ }^{1}$, Qin Jian ${ }^{1}$ \\ ${ }^{1}$ College of Information and Electrical Engineering, China Agricultural University, Beijing, \\ P. R. China 100083 \\ * Corresponding author, Address: College of Information and Electrical Engineering, China \\ Agricultural University, Beijing 100083, P. R. China, Tel: 13621097034, Email: \\ lilincau@gmail.com
}

\begin{abstract}
It is an important to offer a user friendly interface in WebGIS. Excellent map services can be offered by ArcGIS Server. But the problem that the client fetches some data on a Web page from the server quickly is not solved well by ArcGIS Server, Ajax technology can overcome it. When getting large amount of data from server, It takes long time to parse the data for Ajax. To the study "Beijing Age-old Trees Management System", the paper gives methods to solve two problems. Experiments show that the methods are feasible.
\end{abstract}

Keywords: ArcGIS Server, Ajax, WebGIS

\section{INTRODUCTION}

With the development of Internet, WebGIS plays an important role in people's lives. At present there are many WebGIS development platforms, such as SuperMap IS.net, ArcIMS and ArcGIS Server. ArcGIS Server is a new product of ESRI. It is a platform for building enterprise geographic information system (GIS) applications. It is powerful and it is easy to use.

As large amount of map data, there is the problem that transmits slowly on the Internet. Specially, when client requests information to server and get partial refresh page, server will transmit the whole HTML document of the page to client, it increases the response time. Ajax can solve the problem well. But when getting large amount of data from server, it takes long time

Please use the following format when citing this chapter:

Lei, X., Lin, L., Wang, L. and Jian, Q., 2009, in IFIP International Federation for Information Processing, Volume 293, Computer and Computing Technologies in Agriculture II, Volume 1, eds. D. Li, Z. Chunjiang, (Boston: Springer), pp. 299-305. 
to parse the data for Ajax. The paper studies solutions of the two problems and tests them in the "Beijing Age-old Trees Management System". Experiments show that the methods are feasible.

\section{KEY TECHNOLOGIES}

\subsection{ArcGIS Server}

ArcGIS Server is a platform for building enterprise geographic information system (GIS) applications that are centrally managed, support multiple users, include advanced GIS functionality, and are built using industry standards. (ArcGIS Server Administrator and Developer Guide) Compared to other WebGIS develop platforms ArcGIS Server has some key features:

a. Standard GIS framework

ArcGIS Server provides a standard framework for developing GIS server applications. Its rich functionality allows developers to concentrate on solving organizational problems, not building GIS functionality from scratch.

b. Cost-effective deployment

ArcGIS Server supports enterprise applications, such as Web applications, running on servers, and supporting many users. The ADF runtime is not licensed. This allows multiple server applications to run on multiple Web servers.

c. Web controls

ArcGIS Server provides a set of Web controls. These Web controls simplify the programming model for including mapping functionality in your Web application, and allow developers to focus on more advanced GIS functionality aspects of their applications.

d. Web application templates

ArcGIS Server provides a set of Web application templates as a starting point for developers who want to build Web applications using the Web controls, and as an example of how to use the Web controls to build Web applications.

e. Cross-platform functionality

The ArcGIS Server ADF for Java runs on a variety of UNIX platforms and supports numerous Web servers. The GIS Server itself is supported on Windows, Sun Solaris, and Red Hat Linux. The ADF for .NET is available on a number of Windows platforms.

f. Cross-developer languages 
ArcGIS Server supports a variety of developer languages for its use. This allows the objects to be programmed using a wide range of tools and should not require your programming staff to learn a new or proprietary language.

\subsection{Ajax}

Ajax (Asynchronous JavaScript and XML), is a group of inter-related web development techniques used for creating interactive web applications. (Wikipedia, the free encyclopedia) A primary characteristic is the increased responsiveness and interactivity of web pages achieved by exchanging small amounts of data with the server "behind the scenes" so that entire web pages do not have to be reloaded each time there is a need to fetch data from the server. This is intended to increase the web page's interactivity, speed, functionality and usability.

Ajax is asynchronous, in that extra data is requested from the server and loaded in the background without interfering with the display and behavior of the existing page. JavaScript is the scripting language in which Ajax function calls are usually made. Data is retrieved using the XMLHttpRequest object that is available to scripting languages run in modern browsers, or, alternatively, through the use of Remote Scripting in browsers that do not support XMLHttpRequest. In any case, it is not required that the asynchronous content be formatted in XML. So Ajax plays an important role in WebGIS.

\section{PARTIAL REFRESH PAGES}

Beijing is an ancient city; there are a number of old trees in Beijing. For visual managing and displaying on Internet, the frame of "Beijing Old-age trees management System" is as follow (Fig.1).

When client requests information to server and get partial refresh page, server will transmit the whole HTML document of the page to client, it increases the response time. For example, after user chooses query conditions and clicks "Query" button (it is showed in Fig.2), the entire page is reloaded, but some data are as same as before. It is not necessary for the data to be reloaded, it takes extra time. User hopes to get some data that are refreshed, for instance old-age trees information is showed in Fig.3, the others are not changed. 


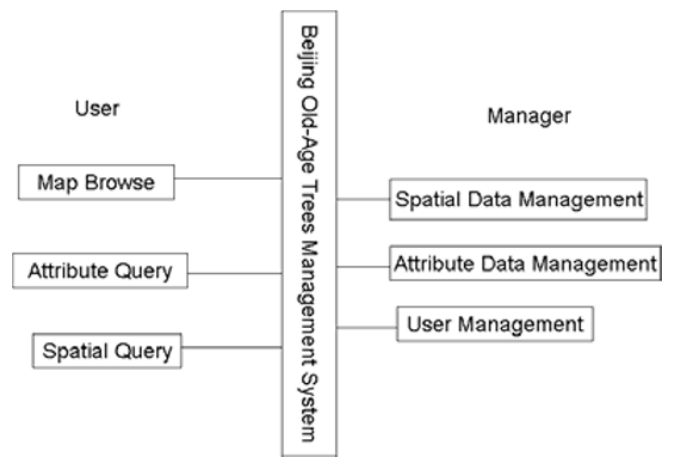

Fig 1. The frame of "Beijing Old-age trees management System"

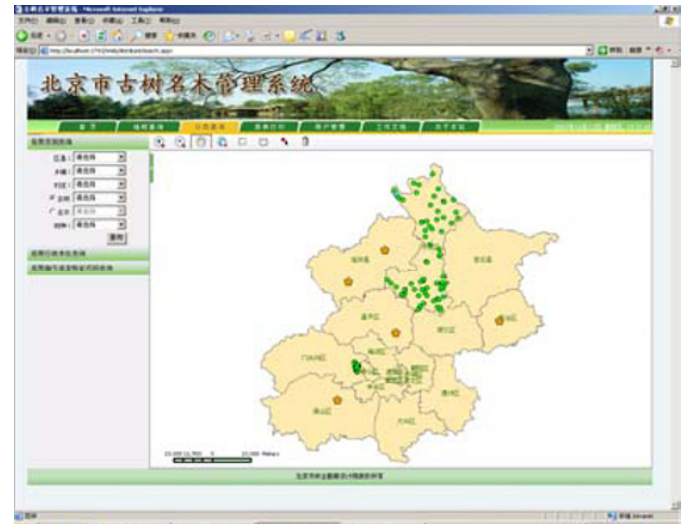

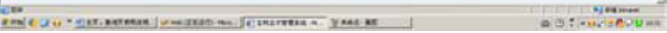

Fig 2. A querying page of "Beijing Old-age trees management System"

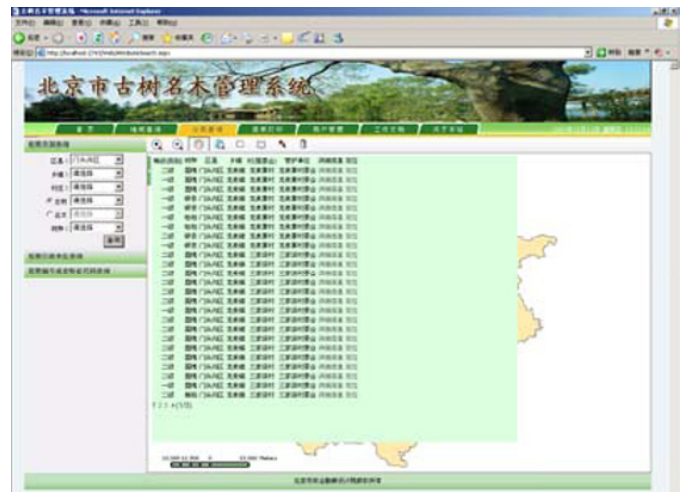

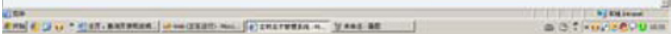

Fig 3. Querying results of "Beijing Old-age trees management System" 
In order to solve the problem we apply AjaxPro.2.dll. AjaxPro.2.dll is the first free libraries which supports visiting servers by a variety of ways. We must follow three steps:

Step 1. Marking "[AjaxPro.AjaxMethod]" in front of calling database method. As follow:

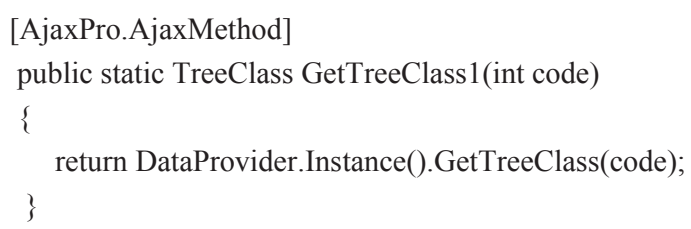

Step 2. Adding following code in the configuration file:

$<$ httpHandlers $>$

$<$ add verb="POST,GET" path="ajaxpro/*.ashx" type="AjaxPro.AjaxHandlerFactory, AjaxPro.2"/>

$<$ httpHandlers $>$

The purpose to adding above code in the configuration file is to ensure that the request of client (Post and GET) can be intercepted by AjaxPro.AjaxhandleFactory.

Step 3. Adding following code while initializing page:

AjaxPro.Utility.RegisterTypeForAjax(typeof(the class name which contains the mothed))

The code can register the calling database method to the client so that the method can be visited at client.

\section{HOW TO ENHANCE SHOWING SPEED}

There are nearly 40,000 records in Beijing Old-age Management System database. Every query may refer hundreds or thousands records. So when the number of result data is large, it is a problem for the system to show the result quickly. Table1 shows the response time of the system when the query refers different number of records.

Table1. Query response time of the system by different number of records

\begin{tabular}{cc}
\hline Records Number & Response Time \\
\hline 800 & $2 \mathrm{~s}$ \\
5000 & $18 \mathrm{~min}$ \\
\hline
\end{tabular}

As Table 1 shows, when there are 800 records needed to be showed at client, it takes $2 \mathrm{~s}$, but when the records number increases to 5000, it takes 18 minutes. The big difference of the response time is because using Ajax.

The principle of Ajax is adding a middle layer called "Ajax Engine" between the client and the server. In this mode, not all the user requests are submitted to the server, only part of requests which really need server to 
complete are sent to the server by the engine. The main functions of the engine include: capture browser events, initialize XMLHttpRequest object, send requests to the server, receive server response, and update client page. The information returned from "Ajax Engine" is normally formatted as text or XML. In our system most information uses XML to transmit data. So all the records returned from server are firstly formatted as XML, and then parse them at client. When the records number is low, it will take little time to format and parse. When data increase to a certain value, it will take huge system resources and time to convert and parse. It is why it takes 18 minutes to show 5000 records.

In order to reduce the response time we take two methods. One is reducing the information returned to the client; the other is that client's showing program is implemented at server and server sends the result of the program running. Table 2 shows the response time to display the same 5000 records.

Table2. Query response time on the two methods

\begin{tabular}{lc}
\hline \multicolumn{1}{c}{ Methods } & Response Time \\
\hline Reducing the information & $4 \mathrm{~min}$ \\
Writing displaying codes in server side & $4 \mathrm{~s}$ \\
\hline
\end{tabular}

Table 2 shows, reducing data amount can save a lot of time in showing data at client. Formerly we sent all the fields of a record back to the client, it takes 18 minutes, but now we only sent two fields back, it takes 4 minutes. It because when the system makes a XML sequence it must convert every field to a XML tag. After the client receiving the XML, firstly it explains all the $\mathrm{XML}$ tags, and then fetching the contents from the tags, finally it puts the contents into a string which HTML can recognize it. If there are many tags it will spend more time to make the string.

But there are two disadvantages in the method. One is it still takes 4 minutes to show data, the other is it losts some useful information. The other method is that client's showing program is implemented at server and server sends the result of the program running. Table 2 shows that it only uses 4 seconds to show 5000 records. But there is a disadvantage in this method: when the user amount goes up, the weight of the server is increasing. So we must use different methods in different cases.

\section{CONCLUSION}

It is important to offer a user friendly interface in WebGIS. The paper discusses two problems. One is refreshing page partly; the other is how to enhance showing speed. It gives solutions of the problems and tests them in 
"Beijing Old-age trees management System". Experiments show that the methods are feasible.

\section{REFERENCES}

Ajax (programming). http://en.wikipedia.org/wiki/AJAX. 2008.

ArcGIS Server Administrator and Developer Guide.

http://www.adaptivepath.com/ideas/essays/archives/000385.php. 2005.

Jesse James Garrett. Ajax: A New Approach to Web Applications.

Jia Qinglei. Knowing ArcGIS Server. China Communication. (in Chinese)

Kang Ling, Fu Junfeng, Wang Huaiqing, Cai Jinsong. Development of WebGIS Based on ArcGIS Server. Water Resources and Power. 2007, 25(1): 26-29.(in Chinese)

Zhao Zili, Wang Donghua, Zhou Xiaoguang. The Exploitation of WebGIS Based on . Net ,ArcObjects And ArcGIS Server. Yao Gan Xin Xi. 2007,(1): 76-80. (in Chinese) 